\title{
Quad Channel Blocker Array Based on a Commodity Liquid Crystal Display
}

\author{
Bernhard Schrenk, Member, IEEE, Paraskevas Bakopoulos, Member, IEEE, and Hannes Hübel
}

\begin{abstract}
We demonstrate channel selection and power leveling based on an off-the-shelf liquid crystal display, addressing datacenter cost targets. An extinction ratio $>10 \mathrm{~dB}$ is reported for a $16 \mathrm{~nm}$ spectral region. Evaluation in a $4 \times 1$ switch configuration confirms colorless channel blocking in presence of side-channels.
\end{abstract}

Index Terms-Optical switches, Optical interconnections, Spatial light modulators, Liquid-crystal devices.

\section{INTRODUCTION}

$\mathrm{T}$ HE enormous growth of datacenter traffic is stimulating interest in hybrid optical switching, as a path towards scaling network capacity beyond the trajectory of Moore's law that is governing the speed of electrical switches. These architectures rely on optical circuit switches for handling longlived ("elephant") flows, while electrical packet switches serve dynamic, short-lived flows (i.e. "mice") [1]. The broad uptake of such concepts is strongly dependent on the cost of the associated photonic components, which need to meet the stringent cost requirements of the datacenter ecosystem. Simple switching elements such as channel blockers working in tandem with wavelength-selective or mode-selective elements are particularly interesting due to their potential for implementing low-cost switching fabrics [2, 3]. Liquid Crystal on Silicon (LCoS) technology as one technological candidate offers low losses, with the added functionality of flexible bandwidth allocation of the blocked channels $[4,5]$, which can be beneficial in some networking concepts.

In this work we demonstrate a low-cost optical switch that relies on a commodity $128 \times 64$ pixels liquid-crystal display (LCD) serving as amplitude-only spatial light modulator (SLM). The LCD switch is combined with passive optical elements to demonstrate a four-element channel blocker array. Despite the use of off-the-shelf visible-light display technology, the device exhibits extinction ratio in excess of 10 $\mathrm{dB}$ and insertion losses of $<12 \mathrm{~dB}$ for a $16-\mathrm{nm}$ spectral region.

Manuscript received March 12, 2017. This work was supported in part by the European Commission through the FP7 Marie Curie Career Integration Grant WARP-5 under Grant $\mathrm{n}^{\circ} 333806$ and through Horizon 2020 research and innovation program under grant agreement $n^{\circ} 645212$ (NEPHELE).

B. Schrenk and H. Hübel are with the Austrian Institute of Technology, Center for Digital Safety\&Security, Donau-City Strasse 1, 1220 Vienna, Austria (phone: +43 50550-4131; e-mail: bernhard.schrenk@ait.ac.at).

P. Bakopoulos is with the School of Electrical \& Computer Engineering, National Technical University of Athens, Iroon Polytechniou 9, 15780 Athens, Greece.
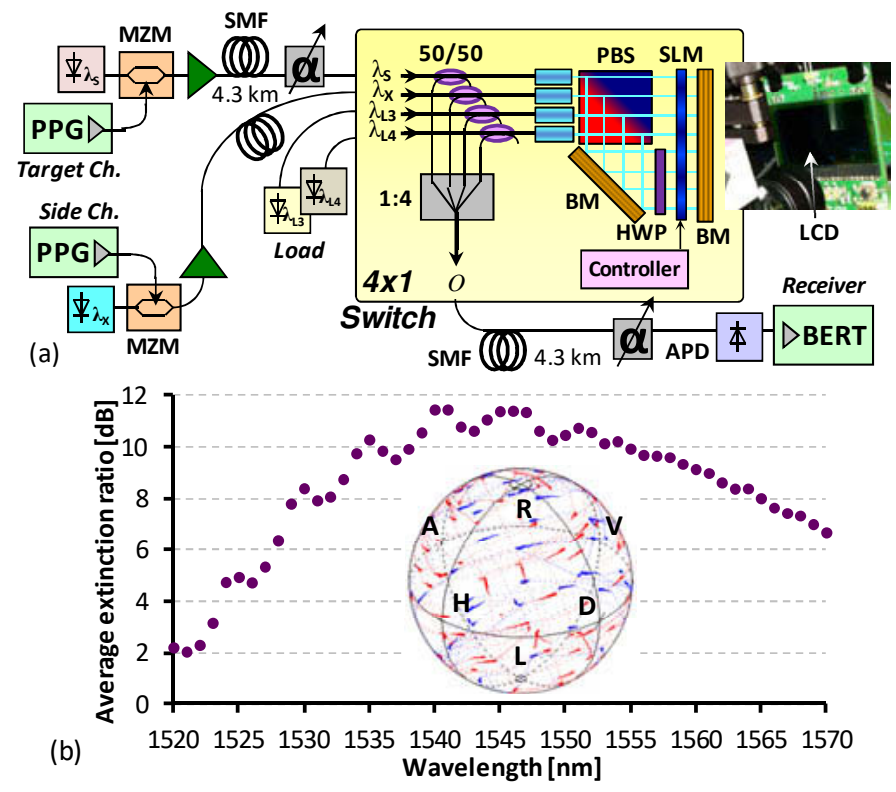

Fig. 1. (a) Architecture of the optical switching node based on commercialoff-the-shelf LCD technology. (b) Spectral dependence of extinction ratio when suppressing optical input signals with the LCD-based blocker.

\section{Characterization OF THE LOW-COST SWITCHING NODE}

The architecture of the proposed channel blocker quad array is presented in Fig. 1(a). It is based on a low-cost commercial off-the-shelf $128 \times 64$ pixels LCD serving as spatial modulator. The signals of four input fibers have been projected with beam collimators in a polarization diversity scheme on the transmissive SLM in order to pass or extinct the corresponding channels. This requires a polarization beam splitter (PBS), broadband mirrors (BM) and a half-wave plate (HWP). The reflective switching engine is completed by 50/50 couplers at the output ports and a 1:4 combiner serving as summing point towards the switch output $O$. The passive splitting losses of this node architecture can be overcome by applying alternative signal combiners, such as WDM multiplexers or photonic lanterns. The power consumption of the device, which also includes a controller for the purpose of SLM reconfiguration, is as low as $0.9 \mathrm{~mW}$, corresponding to $225 \mu \mathrm{W}$ per port.

The channel blocker quad array is configured to implement a switching node situated between multiple transmitters and a receiver and serves to select and forward one incident optical channel. The target and side-channels at $\lambda_{\mathrm{S}}=1550.12 \mathrm{~nm}$ and $\lambda_{\mathrm{X}}=1547.72 \mathrm{~nm}$ are modulated with a PRBS $2^{11}-1$ at $10 \mathrm{~Gb} / \mathrm{s}$ and each launched with $6 \mathrm{dBm}$. Two unmodulated load channels are further added at $\lambda_{\mathrm{L} 3,4}=1548.51 / 1549.32 \mathrm{~nm}$. 

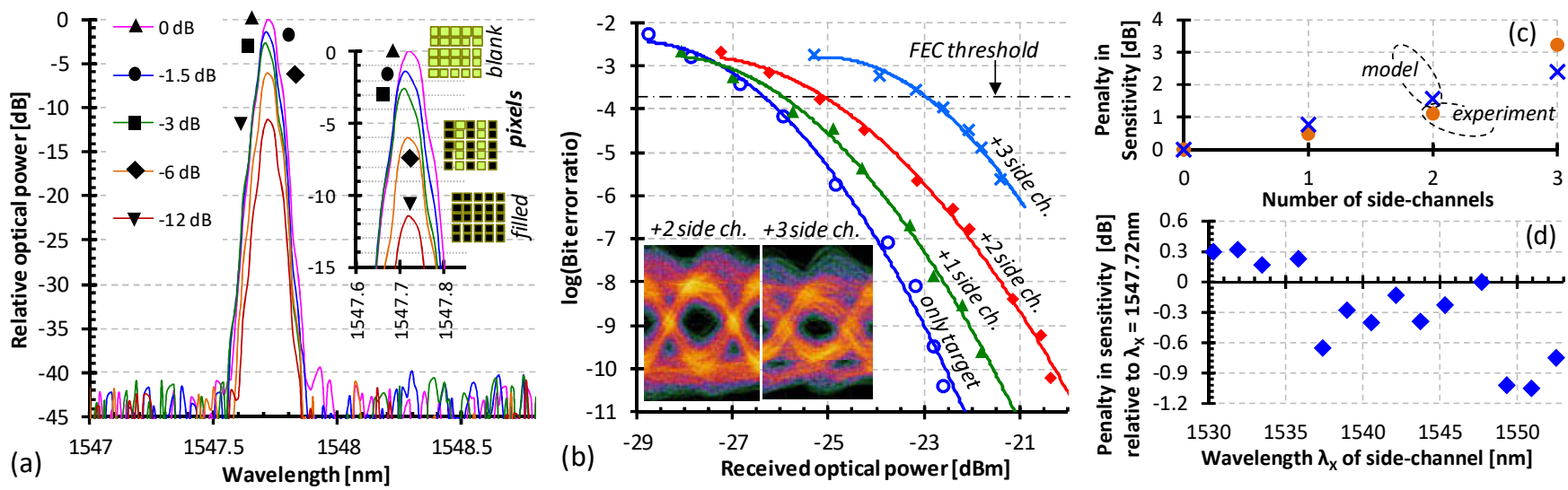

Fig. 2. (a) Power leveling of a signal. (b) BER in presence of side-channels and (c) resulting penalties. (d) Penalty as function of side-channel wavelength.

Transmitters and filterless, APD-based receiver are connected through a $4.3 \mathrm{~km}$ single-mode fiber (SMF) span.

A characterization of the switching node in terms of signal extinction ratio is presented in Fig. 1(b). Measurements have been averaged for polarization swept light states as indicated in the Poincare sphere. An extinction ratio of more than $10 \mathrm{~dB}$ can be obtained over a spectral bandwidth of $16 \mathrm{~nm}$. This is remarkable considering the use of commercial off-the-shelf (COTS) visible-light display technology which is not optimized for telecom applications.

\section{CHANNEL PICKING AND TRANSMISSION PERFORMANCE}

With the ability of the switching node to perform spatial processing of the signals by virtue of the high pixel resolution of the SLM, channel selection and power leveling can be facilitated. The latter is enabled by attributing amplitude masks comprising multiple pixels per light beam and is demonstrated in Fig. 2(a) for the side-channel at $\lambda_{X}$ for a set leveling of $0(\mathbf{\Lambda}),-1.5(\bullet),-3(\mathbf{\bullet}),-6(\bullet)$ and $-12 \mathrm{~dB}(\boldsymbol{\nabla})$ relative to the maximum transmission level.

The transmission performance was evaluated in terms of BER measurements as function of the received optical power for the target channel (Fig. 2(b)). The power level of the sidechannel $\lambda_{X}$ was further increased with respect to the target channel $\lambda_{\mathrm{S}}$ in order to emulate crosstalk from multiple modulated side-channels. BER measurements were conducted under the worst-case polarization state for which bleedthrough of the side-channel and therefore resulting crosstalk is maximized. With reference to transmitting only the target channel (O) the penalty for a present yet suppressed single side-channel ( $\mathbf{\Lambda})$ amounts to $0.9 \mathrm{~dB}$ at a BER of $10^{-10}$. This penalty can be absorbed by the power margin of the link. It has to be noted that no transmission can be established when the side-channel is not suppressed (i.e., passed) by the switch due to the filterless receiver configuration.

In order to investigate scalability for up to 4 channels per node, the power level of the side-channel $\lambda_{X}$ has been increased by 3 and $4.8 \mathrm{~dB}$ to account for 2 and 3 sidechannels, respectively. The penalty in presence of crosstalk arising due to 2 equivalent side-channels ( $)$ with respect to transmission without side-channel $(0)$ is $2.4 \mathrm{~dB}$ at a BER of $10^{-10}$. In case of 3 equivalent side-channels $(\times)$, the penalty increases by $2.2 \mathrm{~dB}$ at the hard-decision Reed-Solomon
$(255,239)$ FEC threshold of $2 \times 10^{-4}$. The observed experimental penalties for adding side-channels are summarized in Fig. 2(c) in reference to the FEC level. Comparison is made with a model based on a reduced eye opening due to finite extinction at the switching node [6]. In this model the suppressed side-channel(s) reside with a small, residual modulation index that reduces the effective modulation extinction ratio $\mathrm{ER}_{\mathrm{S}}$ of the target channel. The resulting penalty in reception sensitivity is then given by 10. $\log \left\{\left(1+1 / E R_{S}\right) /\left(1-1 / E R_{S}\right)\right\}$.

Finally the wavelength dependence of the reception sensitivity of the target channel at $\lambda_{S}=1550.12 \mathrm{~nm}$ on the suppressed yet crosstalk-inducing side-channel at wavelength $\lambda_{X}$ has been investigated. The variation in penalty at the FEC level with reference to the initial case with $\lambda_{X}=1547.72 \mathrm{~nm}$ is presented in Fig. 2(d). A rather small deviation of $<1 \mathrm{~dB}$ has been experienced. This proves the colorless operation.

\section{CONCLUSION}

A $4 \times$ channel blocker based on commodity LCD technology for spatial light selection has been presented. An extinction ratio of $>10 \mathrm{~dB}$ has been obtained over a bandwidth of $16 \mathrm{~nm}$. Transmission in presence of three blocked side-channels and colorless operation within the spectral region of high extinction has been demonstrated.

\section{REFERENCES}

[1] N. Farrington, G. Porter, Y. Fainman, G. Papen, A. Vahdat, "Hunting Mice with Microsecond Circuit Switches", in Proc. ACM HotNets-XI, Redmond, United States, 2012, pp. 115-120.

[2] N.K. Fontaine, R. Ryf, B. Guan, D.T. Neilson, "Wavelength Blocker for Few-Mode-Fiber Space-Division Multiplexed Systems", in Proc. Opt. Fiber Comm. Conf., Anaheim, United States, Mar. 2013, OTh1B.1.

[3] G. de Valicourt et al., "Monolithic Integrated Slot-Blocker for High Datarate Coherent Optical Slot Switched Networks" IEEE/OSA J. Lightwave Technol., vol. 34, no. 8, pp. 1807, Apr. 2016.

[4] Y. Sakurai et al., "LCOS-based wavelength blocker array with channelby-channel variable center wavelength and bandwidth", IEEE Phot. Technol. Lett., vol. 23, no. 14, pp. 989-991, Jul. 2011.

[5] H. Chen, N.K. Fontaine, B. Huang, X. Xiao, R. Ryf, D.T. Neilson, "Wavelength Selective Switch for Dynamic VCSEL-Based Data Centers", in Proc. Europ. Conf. Opt. Comm., Dusseldorf, Germany, Sept. 2016, Th.3.B.1.

[6] B. Schrenk et al., "An All-Optical Carrier Recovery Scheme for Access Networks With Simple ASK Modulation", IEEE/OSA J. Opt. Comm. and Netw., vol. 3, no. 9, pp. 704-712, Sept. 2011. 